\title{
The Geometry of Neutral Paths
}

\author{
ANDREW F. BENNETT \\ College of Earth, Ocean and Atmospheric Sciences, Oregon State University, Corvallis, Oregon
}

(Manuscript received 23 February 2019, in final form 19 August 2019)

\begin{abstract}
Carathéodory's axiomatic development of thermodynamics is applied here to the thermohaline circulation of the ocean. The helicity of the differential form $\not D$ for the density change in an isentropic and isohaline ascent, relative to the change in the water column, does not vanish since the ratio of the thermal and haline compressibilities is a function of pressure. The form for relative density consequently lacks an integrating denominator and so there are no surfaces of constant relative density, or so-called neutral surfaces (McDougall and Jackett). As a consequence of a remarkable theorem (Carathéodory), any two points in the thermohaline state space or equivalently in the real space of the ocean are mutually accessible, in the sense that they can be joined by a neutral path. Many of the paths between any two points and possibly all may only be piecewise smooth. The theorem is supported here with analytical examples of neutral paths in state space, and a numerical example of an idealized ocean in real space, for all of which the seawater obeys a relatively simple equation of state. The existence of multiple neutral paths for pairs of close points is explicitly demonstrated. Some such neutral paths take large excursions throughout state space and throughout the ocean basin. The implications for hydrography and for ocean modeling are discussed.
\end{abstract}

\section{Introduction}

Dynamically defined surfaces such as those of constant potential density have long been used for the synthesis of thermohaline data and for ocean modeling. If there were a state variable of "neutral density," denoted $D$, such that a water parcel ascending with constant entropy and constant salinity experiences a change $d D$ in density relative to the water column, then surfaces of constant $D$ would be ideal for investigations of small-scale mixing (McDougall 1987). Owing to the particular functional dependences of the thermal and haline expansion coefficients as well-defined state variables, the change in relative density is however dependent upon the changes of entropy and salinity in the water column in such a way that the relative density change is not also that of a well-defined state variable (McDougall and Jackett 1988; Klocker and McDougall 2010). In mathematical terms, the differential form for the change is not "perfect" (Chandrasekhar 1939). The form is accordingly denoted $\not D$. It is possible to define "neutral directions" at each thermohaline state, or equivalently at each point in the ocean, such that changes of

Corresponding author: Andrew F. Bennett, bennetan@ oregonstate.edu state or of position in those directions leads to $\not D=0$. If there were an "integrating denominator," or state variable $w$ such that $\not D / w$ is the change $d \phi$ in a well-defined state variable $\phi$, then the neutral directions would everywhere be tangential to surfaces of constant $\phi$ and the latter would suffice for mixing investigations. Here also, owing to the pressure dependence of the ratio of the thermal and haline expansion coefficients, there can be no integrating denominator for the imperfect differential form.

The form does define neutral paths that are everywhere in a local neutral direction, and along which there is no change in relative density. The geometry of these paths is the subject of this study. Much light is shed by Carathéodory's axiomatic development of thermodynamics. He stated and proved an extraordinary theorem concerning any differential form (Carathéodory 1909). The theorem has direct application to the second law of thermodynamics. The objective of this investigation is an application of the theorem to the differential form for relative density changes. The immediate conclusion is that any two neighboring points in the ocean can be connected by a neutral path, that is, the points are mutually "accessible." Accessibility may in practice extend to all of the ocean. The conclusion applies in the thermohaline state space of salinity, potential temperature, and pressure, and also in the real space of the ocean. 
The investigation here commences with introductions of the differential form for relative density and the corresponding neutral paths both in state space and real space. Carathéodory's theorem is then stated. Two theorems by Buchdahl (1966) yield the contrapositive. No proofs are given here. An aspect of particular importance in both proofs is that the differential form is assumed to be defined over all space, be it state space or real space, and that the paths may pass through any part of space. There are bounds on space here, and some pairs of points may only be mutually accessible along bounded paths that are only piecewise smooth. That is, they are "zig-zag" paths. There is no abrupt change of flow direction along such a path, since a neutral path need not be a particle path. A simplification of the oceanic equation of state developed by McDougall (1987) is then used here to construct explicit examples of neutral paths displaying all these findings in state space. A numerical example in real space illustrates the nonuniqueness of neutral paths between a pair of points.

\section{Oceanography}

\section{a. Density}

Notwithstanding the complexity of the physics of liquids and the complexity of the chemistry and biology of seawater, it is assumed here that at equilibrium the substance is characterized by three independent state variables. They are the salinity $S$, the potential temperature $\theta$ with respect to some reference pressure $p_{r}$, and the pressure $p$. The density $\rho$ of seawater is also a state variable, related to the three independent state variables by an equation of state of the form $\rho=\rho(S, \theta, p)$. The state is denoted subsequently by $\mathbf{S}=(S, \theta, p)$.

In the shallow Boussinesq approximation, where infinitesimal density changes $d \rho$ depend linearly upon infinitesimal state changes $d \mathbf{S}$, a water parcel making an infinitesimal adiabatic and isohaline ascent $d z$ within a water column has a dimensionless change in density relative to the water column given by (Gill 1982; McDougall 1987)

$$
\not D \equiv g^{-1} \mathscr{N}^{2} d z=\alpha d \theta-\beta d S,
$$

where $\mathscr{N}$ is the Brunt-Väisälä or buoyancy frequency, $d \theta$ and $d S$ are the changes in the thermohaline profiles within the column, while the thermal expansion coefficient $\alpha$ and haline expansion coefficient $\beta$ are

$$
\alpha=-\frac{1}{\rho}\left(\frac{\partial \rho}{\partial \theta}\right)_{S p}, \quad \beta=\frac{1}{\rho}\left(\frac{\partial \rho}{\partial S}\right)_{\theta p} .
$$

See McDougall (1987) for the relation of $\alpha$ and $\beta$ to expansion coefficients with respect to $(S, T, p)$, where $T$ is the in situ temperature. The middle expression in (1) is the negative of the dimensionless vertical acceleration associated with a small oscillation of amplitude $d z$ within the water column. As remarked in McDougall (1987), (1) is in effect a formula for the local buoyancy frequency:

$$
\mathscr{N}^{2}=g\left(\alpha \frac{\partial \theta}{\partial z}-\beta \frac{\partial S}{\partial z}\right) .
$$

The right hand side of (1) can be evaluated for any changes $d \mathbf{S} \equiv(d S, d \theta, d p)$, which need not be found in any water column. In particular, it will be seen below that the right hand side can be evaluated for the change $d \mathbf{S}$ arising from a displacement $d \mathbf{x}=(d x, d y, d z)$ in a smooth thermohaline field $\mathbf{S}(\mathbf{x})$ over the real space $\mathbf{x}=$ $(x, y, z)$. It is to be emphasized that the differential form (1) is three-dimensional, since $\alpha$ and $\beta$ do depend upon the pressure $p$. The form is denoted subsequently by $\not d D=\mathbf{s} \cdot d \mathbf{S}$, where $\mathbf{s} \equiv(-\beta, \alpha, 0)$. The symbol $\not d$ indicates that the differential $\not d D$ is not perfect, in the sense that there is no state variable $D(S, \theta, p)$ such that $\nabla D=\mathbf{s}$. There may, again, be an integrating denominator $w(S, \theta, p)$ such that $d D / w$ is perfect. There would then be a state variable or "potential" $\phi$ such that $d \phi=\not d / w$. Any function of $w$ is also an integrating denominator for $\not D$. An integrating denominator must satisfy

$$
\nabla w \times \mathbf{s}=w \nabla \times \mathbf{s} .
$$

Theorem 1: The linear differential form (1) has an integrating denominator only if the helicity $\chi \equiv$ $\mathbf{s} \cdot \nabla \times \mathbf{s}$ vanishes.

Here $\mathbf{s}=(-\beta, \alpha, 0)$ and so the condition is

$$
\chi=\beta^{2} \frac{d \gamma}{d p}=0,
$$

where $\gamma$ is the ratio $\alpha / \beta$. It does depend on pressure, so the form (1) is three-dimensional and from (5) it follows that $\not D$ has no integrating denominator.

Suppose that the helicity $\chi$ did vanish. Then (4) would be a system of linear equations for the vector $\nabla w$, while the vectors $\mathbf{s}, \nabla \times \mathbf{s}$ and $\mathbf{s} \times \nabla \times \mathbf{s}$ would form an orthogonal basis for $\nabla w$. The solution for the projections of $\nabla w$ in this basis would lead to an infinite sequence of solvability conditions like (4). The difficulty is overcome by integrating $w$ along the characteristics of (4).

Theorem 2 (Chandrasekhar 1939, chapter 1.8): Every two-dimensional differential form has an integrating denominator. 


\section{b. Neutral paths in state space}

Since, again, the helicity $\chi$ does not vanish there can be no surfaces of uniform relative density, or in other words no neutral surfaces. The differential form (1) is nevertheless of great interest. Indeed, consider a path $\mathbf{S}(u)=(S(u), \theta(u), p(u))$, where $u$ is a scalar path parameter in some range. Suppose that $\mathbf{S}(u)$ is a solution of the ordinary differential equation $\alpha \dot{\theta}-\beta \dot{S}=0$. If a small change in state along the solution path is the same as the change in the thermohaline profiles within some water column, owing to a small change in height in the column, then there can be no strictly vertical accelerations at that height in the column. The pair of states $\mathbf{S}_{0}$ at $u=0$ and $\mathbf{S}_{1}$ at $u=1$ are mutually accessible if there is such a solution path or "neutral path" connecting the pair.

Displaying the arguments in detail, a neutral path is subject to the single constraint

$$
\frac{d S}{d u}(u)=\gamma(S(u), \theta(u), p(u)) \frac{d \theta}{d u}(u) .
$$

Both of the functions $\theta(u), p(u)$ in (6) may be freely chosen, so long as they take the terminal values $\theta_{0}$, $p_{0}$ and $\theta_{1}, p_{1}$, respectively. It may be assumed that $\Delta \theta=\theta_{1}-\theta_{0}>0$, since accessibility is mutual. The potential temperature path $\theta(u)$ is now chosen to be monotone increasing. There are other neutral paths for which $\theta(u)$ is not monotone, but the objective here is the construction of at least one neutral path from $\mathbf{S}_{0}$ to $\mathbf{S}_{1}$ as simply as possible. To proceed, the potential temperature is chosen as the path parameter, that is, $u=\theta$. Then (6) becomes

$$
\frac{d S}{d \theta}=\gamma(S(\theta), \theta, p(\theta))
$$

The solution of (7) must take the terminal values $S_{0}$ and $S_{1}$ at $\theta_{0}$ and $\theta_{1}$, respectively. The roles of $S$ and $\theta$ could of course be reversed. The roles of $\theta$ and $p$ could also be reversed, especially since both make only linear contributions to the simplified expression for $\gamma$ used below in section 4 and since the contributions are comparable. Regardless of the choice and so long as there is a path which is entirely smooth, only one free parameter in $p(\theta)$ or in $\theta(p)$ is required in order to gain access from one state to another. It is required that solutions $S(\theta)$ of (7) be continuous. The interpolation $p(\theta)$ must also be continuous, in which case $S(\theta)$ is smooth. The pressure interpolation need only be piecewise smooth. Quadratic interpolation of pressure with respect to $\theta$ and also sinusoidal interpolation have been chosen here for illustrations of access. An empirical polynomial for $\gamma(S, \theta, p)$ has been developed from oceanographic data which include a comprehensive range of observed values (McDougall 1987). The state variable $\gamma$ is positive throughout the range. If it were constant then (7) would permit only the straight line $S(\theta)=S_{0}+\gamma\left(\theta-\theta_{0}\right)$. The empirical formula for $\gamma(S, \theta, p)$ is given, but on a neutral path the pressure $p(\theta)$ is again arbitrary except for its terminal values $p_{0}$ and $p_{1}$ respectively at $\theta_{0}$ and $\theta_{1}$, while $S(\theta)$ is apparently overdetermined by (7) and its terminal values $S_{0}$ and $S_{1}$. Again, it is required that $S\left(\theta_{0}\right)=S_{0}$ and the on-path pressure $p(\theta)$ must be chosen so that $S\left(\theta_{1}\right)=S_{1}$.

\section{c. Neutral paths in real space}

Let $\mathbf{x}=(x, y, z)$ denote points in real space, and consider thermohaline fields $\mathbf{S}(\mathbf{x})$ that are smooth over real space. A small neighborhood in real space is associated with a small neighborhood in state space and vice versa provided the Jacobian determinant $J=$ $\operatorname{det}[\mathbf{J}]$, where $\mathbf{J}(\mathbf{x})$ is the Jacobian matrix for $\mathbf{S}(\mathbf{x})$, does not vanish locally. It is readily seen that $J=\nabla S \cdot \nabla \theta \times$ $\nabla p$. The differential form (1) has a real space image, that is, $\not D=\mathbf{X} \cdot d \mathbf{x}$ where $\mathbf{X}=\mathbf{s} \cdot \mathbf{J}=-\beta \nabla S+\alpha \nabla \theta$.

It unfortunate that the roles of upper and lower case letters in real space are the opposite of their roles in real space, but the above notation for $\not D$ in real space is that of Bernstein (1960), Buchdahl (1966), Carathéodory (1909), and Pogliani and Berberan-Santos (2000). The notation of Zachanoglou (1973) is $\mathbf{a} \cdot d \mathbf{x}$, which would create other conflicts here. The introduction of yet another notation for real space would be more confusing than following convention. Finally, Gill (1982) denotes salinity by $s$, while McDougall and Feistal (2003) denote potential temperature by $\Theta$.

The helicity $\chi$ transforms as $\chi=\mathbf{s} \cdot \nabla \times \mathbf{s}=J^{-1} \mathbf{X} \cdot \nabla \times$ $\mathbf{X}$. A small neighborhood in state space may in practice be associated with a large region of real space. Equally, a real space neighborhood that includes a near front is by definition associated with a large region in state space.

A neutral path in state space takes, again, the form $\mathbf{S}(u)$ for $u$ in some range. What is sought is the real path $\mathbf{x}(u)$ such that, with extreme notational sloppiness, $\mathbf{S}(u)=\mathbf{S}[\mathbf{x}(u)]$. It follows immediately that

$$
\mathbf{J}(u) \cdot \frac{d \mathbf{x}(u)}{d u}=\frac{d \mathbf{S}(u)}{d u} .
$$

The above is a linear system for $d \mathbf{x}(u) / d u$, and can be solved by Cramer's rule. The solvability condition is $J \neq 0$. Thus the two functional degrees of freedom in $\mathbf{S}(u)$ are also two degrees of freedom in $\mathbf{x}(u)$, subject to the terminal constraints $\mathbf{x}_{0}$ and $\mathbf{x}_{1}$. There may be several pairs of real points corresponding to the same pair of states, but not vice versa. It may be noted that a neutral path encircling an ocean basin can be constructed by integrating the real-space differential equation (8) 
around some closed horizontal path that encircles the basin. The neutral path thus returns to the initial water column, but not necessarily to the initial depth. There is a corresponding neutral path in state space, and in general the state space path is also open.

Again, the ratio $\gamma=\alpha / \beta$ depends upon pressure and so there are no true neutral surfaces. See McDougall and Jackett (1988), Klocker and McDougall (2010), and the references therein. Examples of the construction of "near-neutral surfaces" may be found in McDougall (1987), McDougall and Jackett (1988), Theodorou (1991), and Klocker and McDougall (2010). The examples are based on hydrographic surveys, which are available in gridded formats. The following discussion assumes, for simplicity, that the surveys are smooth functions of continuously varying real position. That is, the surveys consist of the thermohaline fields $(S, \theta, p)$ over a domain of $(x, y, z)$. Discrete summation on a grid is thereby replaced with continuous integration. To paraphrase McDougall (1987, p. 1955), integration originates in the south-west corner of a basin, level by level, and extends along the southernmost latitude, thereby constructing a family of neutral paths occupying the latitudinal southern boundary. Then integrations are performed along longitudes, yielding near-neutral surfaces. Further integrations yield helical surfaces. The helical pitch across major ocean basins is less than about a meter (McDougall and Jackett 1988; Theodorou 1991). The construction does not take into account the possibility that there are many other neutral paths connecting any pair of points chosen on the surface so constructed.

\section{Accessible and inaccessible states}

Any two states $\mathbf{S}_{0}$ and $\mathbf{S}_{1}$ that can be connected by a neutral path are mutually accessible. If there is no such path then they are mutually "inaccessible." The following theorem is elementary.

Theorem 3: If (1) has an integrating denominator, then in any neighborhood of a state $\mathbf{S}_{0}$ there is a state $\mathbf{S}_{1}$ which is inaccessible from $\mathbf{S}_{0}$.

The converse of Theorem 3 is also true.

Theorem 4 (Carathéodory 1909): Consider the differential form (1). If every neighborhood of a state $\mathbf{S}_{0}$ includes a state $\mathbf{S}_{1}$ which is inaccessible from $\mathbf{S}_{0}$ then (1) has an integrating denominator.

The proof is elegant with respect to the conceptual geometry, and also with respect to the complete lack of analytical detail. Solution surfaces are constructed from families of neutral paths on families of cylinders in state space. Closely neighboring states on these cylinders may or may not be mutually accessible, even via long solution paths. The online translation of Carathéodory (1909) by D. H. Delphenich ${ }^{1}$ contains some insignificant typographic errors. There is a significant error, namely, the omission of a negative on page 13 of the translation. The sentence which begins "The points $Q_{i}$ can also be reached by curves" should be "The points $Q_{i}$ cannot also be reached by curves." See Carathéodory (1909, p. 369): "Die Punkte $Q_{i}$ können auch nicht von $P$ aus längs Kurven."

Bernstein (1960) points out that Carathéodry's proof only establishes the local existence of an integrating denominator. That is, for some small $\varepsilon>0$ there is a sphere of radius $\varepsilon$ centered on $\mathbf{S}_{0}$ in which an integrating denominator exists. Carathéodory's proof constructs a potential function, but the potential function is not proved to be smooth in a finite region. The latter is a region with dimensions comparable to the scales of variability of the coefficients in the differential form. Here, however, the interest is in the contrapositive of the theorem. If the helicity does not vanish even locally then an integrating denominator cannot exist even locally, and so according to the contrapositive there must be a neighborhood of $\mathbf{S}_{0}$ in which every $\mathbf{S}_{1}$ is accessible. The examples in section 4 show that if the helicity does not vanish in a large region then the neighborhood of accessibility may be large.

A less well-known monograph on thermodynamics (Buchdahl 1966) includes the following two theorems, with detailed analytical proofs in an $N$-dimensional state space. The first theorem establishes that if $\chi_{N}$, being the $N$-dimensional analog of the helicity $\chi$, vanishes locally then there exists a local integrating denominator. A globally smooth potential function of any two of the state variables is constructed, but it is not proved to be a smooth function in a finite region of the other variables. The case of interest here is a finite region in which the $\chi_{3}$ does not vanish anywhere. The second theorem then establishes the accessibility of any state $\mathbf{S}_{1}$ neighboring $\mathbf{S}_{0}$. A long path joining two neighboring states, as contemplated by Carathéodory, is constructed by Buchdahl in detail. The first theorem and the contrapositive of the second imply Carathéodory's theorem as modified by Bernstein.

\section{Theorem 5 (Buchdahl 1966): If $\chi_{N}=0$ then $\not D$ has an} integrating denominator.

The number of nonvanishing coefficients in any differential form of dimension $N$ may be reduced

\footnotetext{
${ }^{1}$ http://www.neo-classical-physics.info/uploads/3/4/3/6/34363841/ caratheodory_-_thermodynamics.pdf.
} 


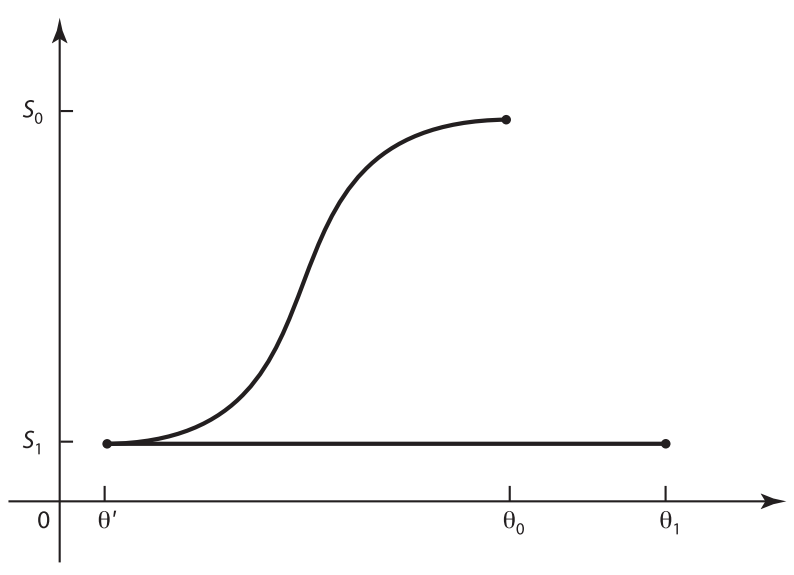

FIG. 1. A piecewise smooth neutral path between states $\mathbf{S}_{0}$ and $\mathbf{S}_{1}$ where $\Delta S<0$. The pressure $p(\theta)$ vanishes between $\mathbf{S}^{\prime}$ and $\mathbf{S}_{1}$, and interpolates quadratically between $\mathbf{S}^{\prime}$ and $\mathbf{S}_{0}$. The salinity $S(\theta)$ is respectively a constant and a cubic.

to two, by repeated construction of analogs of potential temperature. If $\chi_{N}$ vanishes for one value of $N$, it vanishes for all values, and so the final form is then two-dimensional.

Theorem 6 (Buchdahl 1966): If $\chi_{N} \neq 0$ then there is a neighborhood of any point in which every other point is accessible.

Integrating the ordinary differential equation (7) for salinity on a neutral path yields the inequalities

$$
\gamma_{\min } \Delta \theta<\Delta S<\gamma_{\max } \Delta \theta,
$$

since $\gamma$ is positive, since the mutuality of accessibility ensures that $\Delta \theta$ may be assumed to be positive and since monotone interpolation of $\theta$ has been chosen. There can therefore be no neutral path from $\mathbf{S}_{0}$ to $\mathbf{S}_{1}$ if $G \equiv \Delta S / \Delta \theta$ lies outside the interval $\left[\gamma_{\min }, \gamma_{\max }\right]$ of observed values for $\gamma$. The difficulty is resolved by the construction of paths that are continuous but only piecewise smooth. Another state $\mathbf{S}^{\prime}$ is selected so that both $\mathbf{S}_{0}$ and $\mathbf{S}_{1}$ are accessible from $\mathbf{S}^{\prime}$ by paths for which $\Delta \theta$ is positive and $G$ lies within the observed range of values of $\gamma$. The following highly simplified example illustrates the construction, in which the path parameter $u$ is $\theta$.

Consider the simplified ratio $\gamma(S, \theta, p)=c p$ where $c$ is a positive constant. Suppose that $\Delta S=S_{1}-S_{0}<0$, $\Delta \theta=\theta_{1}-\theta_{0}>0$, and $p_{0}=p_{1}=0$. Choose any $\theta^{\prime}$ such that $0<\theta^{\prime}<\theta_{0}$. See Fig. 1 . Then for the range $\theta^{\prime} \leq \theta \leq \theta_{1}$ the pressure $p(\theta)=0$ yields a neutral path between $\mathbf{S}^{\prime}=$ $\left(S_{1}, \theta^{\prime}, 0\right)$ and $\mathbf{S}_{1}=\left(S_{1}, \theta_{1}, 0\right)$. Now choose quadratic interpolation of pressure from $\mathbf{S}^{\prime}$ to $\mathbf{S}_{0}$, that is, choose $p(\theta)=r_{2}\left[\left(\theta-\theta^{\prime}\right)^{2}-\left(\theta-\theta^{\prime}\right)\left(\theta_{0}-\theta^{\prime}\right)\right]$ for the range $\theta^{\prime}<$ $\theta<\theta_{0}$. Pressure is positive and so $r_{2}$ must be negative.
The salinity satisfying (7) is $S(\theta)=S_{1}+c r_{2}\left[\left(\theta-\theta^{\prime}\right)^{3 / 3}-\right.$ $\left.\left(\theta-\theta^{\prime}\right)^{2}\left(\theta_{0}-\theta^{\prime}\right) / 2\right]$. The choice $r_{2}=-6\left(S_{0}-S_{1}\right) / c\left(\theta_{0}-\theta^{\prime}\right)^{3}$ yields a neutral path between $\mathbf{S}^{\prime}$ and $\mathbf{S}_{0}$. There is accordingly at least one continuous but only piecewise smooth neutral path, or "zig-zag" path, between $\mathbf{S}_{0}$ and $\mathbf{S}_{1}$. Again, the bounds upon $G$ are overcome by a path that is only piecewise smooth but which exploits the mutuality of access. A shortcoming of quadratic interpolation of pressure is that it may also go out of bounds. Piecewise linear interpolation of $\theta$ and $p$ assures that both remain in bounds, and assures that $\theta$ is piecewise monotone. If $S_{0}$ and $S_{1}$ are close then it is straightforward to show that a short zig-zag can close the gap.

\section{Illustrations}

\section{a. Chandrasekhar's example}

The first chapter of a celebrated treatise on stellar structure (Chandrasekhar 1939) includes a simple example. The differential form is $\not L=-y d x+x d y+K d z$, where $K$ is a constant. The helicity is $C=2 K$. The two directions $(x, y, 0)$, and $\left(-K y, K x,-r^{2}\right)$ where $r^{2}=x^{2}+y^{2}$, lie in the local tangent plane defined by $\not L$. The first direction leads to the solution paths $\left(x, x \tan \phi_{0}, z_{0}\right)$ where $\tan \phi_{0}=y_{0} / x_{0}$, and $\left(x, x \tan \phi_{1}, z_{1}\right)$ where $\tan \phi_{1}=$ $y_{1} / x_{1}$. The second direction leads to circular helices. The modularity of $\phi$ assures that the spiral radius $r$ may be chosen so that $r^{2}=-K\left(z_{1}-z_{0}\right) /\left(\phi_{1}-\phi_{0}\right)$. That is to say, there is a solution path from $\left(x_{0}, y_{0}, z_{0}\right)$ to $\left(x_{1}, y_{1}, z_{1}\right)$ consisting of a segment of a ray in the plane $z=z_{0}$, a segment of a circular helix passing through that plane and also through the plane $z=z_{1}$, and finally a ray segment in the plane $z=z_{1}$. The construction depends crucially on the assumption that $K \neq 0$. Otherwise the second direction would lead only to "vertical" lines that could not connect the two horizontal rays, and further to the conclusion that the plane $y=x\left(y_{0} / x_{0}\right)$ is a potential surface. It may be noted that neutral helices with vertical axes have been constructed in the real ocean. The pitch across an ocean basin is, again, about a meter (McDougall and Jackett 1988; Theodorou 1991).

Chandrasekhar's example shows that accessibility may not be restricted to a small neighborhood.

\section{b. There may be only one potential surface}

Zachanoglou (1973) provides the curious example

$$
\not L=x z d y+d z .
$$

The helicity $\chi=z$ vanishes on the plane $z=0$, but nowhere off this plane. In other words, while there is a single potential there is no family of potentials. 


\section{c. Linear pressure dependence}

Assume $\gamma=\varepsilon_{0}+\varepsilon_{1} p$, where $\varepsilon_{0}$ and $\varepsilon_{1}$ are constants. Let the path parameter be $\theta$, and let the pressure interpolation be quadratic. That is, let

$$
p(\theta)=p_{0}+r_{1}\left(\theta-\theta_{0}\right)+r_{2}\left(\theta-\theta_{0}\right)^{2},
$$

where $r_{1}$ and $r_{2}$ are as yet undetermined constants subject only to $r_{1} \Delta \theta+r_{2} \Delta \theta^{2}=\Delta p=p_{1}-p_{0}$. An elementary calculation yields

$$
r_{2}=-\frac{6}{\varepsilon_{1} \Delta \theta^{3}}\left\{\Delta S-\Delta \theta\left[\varepsilon_{0}+\varepsilon_{1}\left(p_{0}+\Delta p / 2\right)\right]\right\} .
$$

Hence $\left|r_{2}\right| \rightarrow \infty$ as $\varepsilon_{1} \rightarrow 0$ and so $p(\theta)$ is a high (deep) parabola for $r_{2}<0\left(r_{2}>0\right)$. The neutral path between $\mathbf{S}_{0}$ and $\mathbf{S}_{1}$ is smooth. There are, however, values for $p_{0}, \varepsilon_{0}, \varepsilon_{1}$, and $\Delta \mathbf{S}$ such that $p$ or $\gamma$ becomes negative on the path.

The complete empirical polynomial for $\gamma$ is of the form

$$
\gamma(S, \theta, p)=\gamma_{2}(S-35.0)^{2}+\gamma_{1}(S-35.5)+\gamma_{0},
$$

where $\gamma_{2}=-0.678662 \times 10^{-5}$, while $\gamma_{1}$ and $\gamma_{0}$ are polynomials in $p$ and $\theta$ (McDougall 1987). Thus $\gamma_{1}$ and $\gamma_{0}$ are functions of $\theta$ on a path, and (7) is a Riccati equation that in general can have singular solutions. The asymptotic form of the equation near a singularity is $d R / d \theta \sim \gamma_{2} R^{2}$, where $R=S-35.0$. The general solution near the singularity is

$$
R \sim\left[R_{0}^{-1}-\gamma_{2}\left(\theta-\theta_{0}\right)\right]^{-1} .
$$

The denominator in (14) does not vanish in the state space of the ocean, that is, singular solutions do not occur in the ocean.

The quadratic term in (13) contributes about $1 \%$ of the right hand side, and so with sufficient accuracy for this investigation the neutral paths in general satisfy

$$
\frac{d R}{d \theta}=\gamma_{1} R+\gamma_{0} .
$$

The solution of (15) at $\theta_{1}$ is

$$
R\left(\theta_{1}\right)=R\left(\theta_{0}\right) e^{\Lambda\left(\theta_{1}\right)}+\int_{\theta_{0}}^{\theta_{1}} \gamma_{0}(\theta) \exp \left[\Lambda\left(\theta_{1}\right)-\Lambda(\theta)\right] d \theta
$$

where $\Lambda(\theta)=\int_{\theta_{0}}^{\theta} \gamma_{1}\left(\theta^{\prime}\right) d \theta^{\prime}$. Then the form of the argument $p(\theta)$ in $\gamma_{1}(\theta, p)$ and in $\gamma_{0}(\theta, p)$ is chosen so that $R\left(\theta_{0}\right)=S_{0}-35.0$ and $R\left(\theta_{1}\right)=S_{1}-35.0$. If $p(\theta)$ is smooth then only a single disposable parameter is
TABLE 1. Real terminals.

\begin{tabular}{ccccccc}
\hline \hline Path & $x_{0}$ & $x_{1}$ & $y_{0}$ & $y_{1}$ & $z_{0}$ & $z_{1}$ \\
\hline 1 & $10^{5}$ & $4.9 \times 10^{6}$ & $2.4 \times 10^{6}$ & $1.2 \times 10^{6}$ & $-1.2 \times 10^{3}$ & $-10^{3}$ \\
2 & $10^{5}$ & $4.9 \times 10^{6}$ & $2.4 \times 10^{6}$ & $1.2 \times 10^{6}$ & $-1.2 \times 10^{3}$ & $-10^{3}$ \\
\hline
\end{tabular}

required in order that $S\left(\theta_{1}\right)=S_{1}$. Again a quadratic suffices. Successive corrections in powers of $\gamma_{2}$ may be added to (16) but do not alter the conclusion. Every thermohaline state in the ocean is smoothly accessible from every other state, although the neutral path may enter unrealistic ranges of $p$ or $\gamma$ or even $S$.

\section{d. Two coterminal neutral paths in an idealized ocean}

Explicit solutions for neutral paths in state space and real space may be approximated by restricting the McDougall (1987) formula for $\gamma$ to the linear terms only. The approximation, which numerical integration reveals to be sufficiently accurate for this exploratory investigation, is

$$
\gamma(S, \theta, p)=f+a(S-35.0)+b \theta+c p,
$$

where

$$
\begin{array}{ll}
f=0.665157 \times 10^{-1}, & a=0.378110 \times 10^{-2}, \\
b=0.170907 \times 10^{-1}, & c=0.380374 \times 10^{-4} .
\end{array}
$$

Let the pressure interpolation again be quadratic, as in (11). Elementary calculus yields a solution for the on-path salinity $S(\theta)$. The dependence upon the control parameter $r_{2}$ is linear, and $r_{2}$ is explicitly determined by the requirement that $S\left(\theta_{1}\right)=S_{1}$.

Many other pressure interpolation schemes suffice for the construction of a neutral path from $\mathbf{S}_{0}$ to $\mathbf{S}_{1}$, for example,

$$
p(\theta)=p_{0}+\Delta p \frac{\sin \left[\lambda\left(\theta-\theta_{0}\right)\right]}{\sin (\lambda \Delta \theta)}
$$

There is a corresponding explicit formula for $S(\theta)$. Its dependence on the control parameter $\lambda$ is nonlinear, but $\lambda$ may be precisely estimated by minimizing $\left|S_{1}-S\left(\theta_{1}\right)\right|^{2}$. There is a solution for $\lambda$ such that the minimum vanishes, so long as the range of $S(\theta)$ is continuous and includes the salinities in the ocean region of interest.

While all the smooth interpolation schemes yield smooth paths between a pair of points, the paths may access inadmissible states en route.

\section{e. Neutral paths in real space}

In this illustration the thermohaline fields over real space are idealizations. The spatial domain of the 
TABLE 2. State terminals.

\begin{tabular}{ccccccc}
\hline \hline Path & $S_{0}$ & $S_{1}$ & $\theta_{0}$ & $\theta_{1}$ & $p_{0}$ & $p_{1}$ \\
\hline 1 & 34 & 35.92 & 5 & 10 & 5000 & 5200 \\
2 & 34 & 35.92 & 5 & 10 & 5000 & 5200 \\
\hline
\end{tabular}

Cartesian coordinates $(x, y, z)$ is Basin $=\left[0, x_{e}\right] \times\left[0, y_{n}\right] \times$ $\left[z_{b}, 0\right]$, where $x_{e}=5 \times 10^{6} \mathrm{~m}, y_{n}=5 \times 10^{6} \mathrm{~m}$, and $z_{b}=-10^{4} \mathrm{~m}$. The surface salinity $S$ and surface potential temperature $\theta$ are respectively linear functions of $y$ and $x$, where

$$
\begin{aligned}
& S(x, y, 0)=S_{s}+\left(y / y_{n}\right)\left(S_{n}-S_{s}\right), \\
& \theta(x, y, 0)=\theta_{w}+\left(x / x_{e}\right)\left(\theta_{e}-\theta_{w}\right) .
\end{aligned}
$$

The disposable thermohaline values on the lateral boundaries are $S_{s}, S_{n}, \theta_{w}$, and $\theta_{e}$. The fields of $S$ and $\theta$ decay exponentially in depth, while $p$ in decibars is identified with depth $-z$ in meters. Thus the vertical profiles are

$$
\begin{aligned}
& S(x, y, z)=S_{b}+\exp \left(-z / z_{t}\right)\left[S(x, y, 0)-S_{b}\right], \\
& \theta(x, y, z)=\theta_{b}+\exp \left(-z / z_{t}\right)\left[\theta(x, y, 0)-\theta_{b}\right],
\end{aligned}
$$

where $z_{t}=-10^{3} \mathrm{~m}$ is the thermocline depth scale. The disposable values on the surface at $z=0$ and those on the bottom at $z=z_{b}$ are $S_{f}, \theta_{f}, S_{b}$, and $\theta_{b}$, respectively. The various bounding values in both of the following two examples are

$$
\left(S_{s}, S_{n}, \theta_{w}, \theta_{e}, S_{b}, \theta_{b}, p_{b}\right)=\left(35,25,10,20,30,2,10^{4}\right) .
$$

The salinity $S(\theta)$ on a neutral path constructed in state space is close to monotone as a function of $\theta$, but the zonal coordinate $y(u)$ can fluctuate significantly as a function of the parameter $u$ along a neutral path constructed in real space.

The parameter on two paths 1 and 2 is chosen to be $x$, and so paths not monotone in $x$ are arbitrarily excluded. The vertical coordinate is $z(x)$ which on path 1 is quadratic as in (11), and which on path 2 is sinusoidal as in (20). Thus, it is the zonal coordinate $y(x)$ that is constrained by the condition of neutrality.

The empirical formula for $\gamma(S, \theta, p)$ given in McDougall (1987) is supported by data in the thermohaline subspace
Data $=[25,40] \times[0,40] \times[0,10000]$. By construction $\gamma$ is positive in Data, as is required, and consequently $d S / d \theta$ is positive on any smooth neutral path. The formula may take negative values if extrapolated outside Data. It suffices that paths 1 and 2 be contained in Basin, since the chosen thermohaline fields are monotonic and their boundary values are in Data. The real path terminals $\mathbf{x}_{0}=\left(x_{0}, y_{0}, z_{0}\right)$ and $\mathbf{x}_{1}=\left(x_{1}, y_{1}, z_{1}\right)$ are shown in Table 1 . The state space terminals $\mathbf{S}_{0}=\left(S_{0}, \theta_{0}, p_{0}\right)$ and $\mathbf{S}_{1}=\left(S_{1}, \theta_{1}, p_{1}\right)$ are shown in Table 2. The control Ctrl, which is $r_{2}$ for path 1 and $\lambda$ for path 2 , is shown in Table 3 along with extrema for $y$ and $z$. The paths are shown in Fig. 2. The projection does not make clear that the paths lie on a relatively unconvoluted surface.

It remains finally to verify that a neutral path constructed in real space maps into a neutral path in state space. The comparison is made as follows. First, recall that the thermohaline fields in Basin are as in (21)-(24). Then numerical integration of

$$
\frac{d \mathscr{S}}{d x}=\gamma(\mathscr{S}, \theta, p) \frac{d}{d x} \theta
$$

subject to $\mathscr{S}_{0}=S\left(x_{0}, y_{0}, z_{0}\right)$, and where $\theta$ and $p$ are evaluated on a real neutral path $(x, y(x), z(x))$, yields the salinity $\mathscr{S}(x)$ in state space. It is compared with $S(x, y(x), z(x))$. The calculated discrepancies for each of paths 1 and 2 are about the same as the truncation errors of $O\left[(\delta x)^{2}\right]$ in the centered integration of (26). The parameter $x$ was discretized with 201 points, and so $\delta x=0.005 \times\left(x_{1}-x_{0}\right)$.

\section{Summary and discussion}

The nonvanishing helicity $\chi$ of the differential form $d D$ for relative density precludes the existence of neutral surfaces. As a consequence of Carathéodory's theorem, the nonvanishing helicity ensures that there are countless neutral paths at least between any neighboring two states or between any two neighboring real points. In general, a smooth path may be parameterized as $(S(u), \theta(u), p(u))$ for some scalar parameter $u$, where $\theta(u)$ and $p(u)$ meet the respective terminal values of $\theta$ and $p$ but otherwise are arbitrarily chosen. Only one degree of freedom in $p(u)$ is needed in order that $S(u)$ meet both terminal values of $S$. Thus, to find at least one smooth neutral path it suffices to choose a

TABLE 3. Real minima, maxima, and controls.

\begin{tabular}{lccccc}
\hline \hline Path & $\min (\mathscr{H})$ & $\max (\mathscr{H})$ & $\min (\mathscr{Z})$ & $\max (\mathscr{Z})$ & Ctrl \\
\hline 1 & $1.200 \times 10^{6}$ & $4.008 \times 10^{6}$ & $-2.640 \times 10^{3}$ & $-10^{3}$ & $6.1535 \times 10^{3}$ \\
2 & $1.145 \times 10^{6}$ & $3.114 \times 10^{6}$ & $-2.029 \times 10^{3}$ & -370.9 & 15.9516 \\
\hline
\end{tabular}




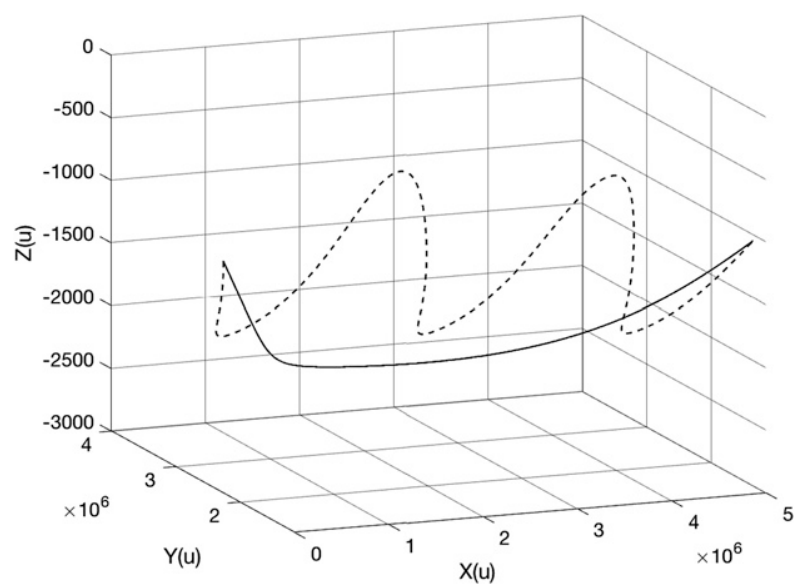

FIG. 2. Path 1, solid line; path 2, dashed line. See Tables 1-3.

monotone form for $\theta(u)$ so that $\theta$ is the path parameter, and to choose $p(u)$ as a quadratic in $\theta$. Many other neutral paths connect the same terminals. For some pairs of states or points a neutral path may only be piecewise smooth. Each neutral path in real space maps into only one in state space, but several in real space may map into the same one in state space.

While there may be near-neutral surfaces for $\not D$, there are many neutral paths between any two points on any such surface and most of the paths do not lie in the surface. Thus, the ideal hydrographic surface for water mass analysis and for ocean modeling remains elusive. On the other hand all the neutral paths at any point osculate the local neutral tangent plane, and so the dynamical significance of the local normal or dianeutral direction remains.

Few modern treatises on thermodynamics mention Carathéodory's axiomatic thermodynamics. The principles of Kelvin and of Clausius are preferred, involving "engines," "reservoirs," and "cycles." See Buchdahl (1966, sections 38 and 50) for a masterful discussion, and see Pogliani and Berberan-Santos (2000) for an historical account. A physicist might first wonder why axiomatic thermodynamics has fallen from favor, and second why should it be favored here. The reasons are first, as argued by Planck, ensuring Carathéodory's assumption is not practicable. Second, it is the contrapositive of Carathéodory's theorem which is of interest here. The known nonvanishing of helicity ensures the negation of Carathéodory's conclusion, and hence the negation of his assumption.

Hydrographic charts of state variables on surfaces of constant salinity or on surfaces of potential density, for examples, would seem in themselves to be unremarkable dynamical constructions. Carathéodory's theorem establishes that the charts imply the existence of a remarkable geometry of neutral paths, with countless neutral paths between any two water masses.

Acknowledgments. Ed Zaron and two anonymous reviewers made constructive comments.

\section{REFERENCES}

Bernstein, B., 1960: Proof of Carathéodory's local theorem and its global application to thermostatics. J. Math. Phys., 1, 222-224, https://doi.org/10.1063/1.1703655.

Buchdahl, H. A., 1966: The Concepts of Classical Thermodynamics. Cambridge University Press, 236 pp.

Carathéodory, C., 1909: Untersuchungen über die Grundlagen der Thermodynamik. Math. Ann., 67, 355-386, https://doi.org/ 10.1007/BF01450409.

Chandrasekhar, S., 1939: An Introduction to the Study of Stellar Structure. Dover, $512 \mathrm{pp}$.

Gill, A. E., 1982: Atmosphere-Ocean Dynamics. International Geophysics Series, Vol. 30, Academic Press, 662 pp.

Klocker, A., and T. J. McDougall, 2010: Quantifying the consequences of the ill-defined nature of neutral surfaces. J. Phys. Oceanogr., 40, 1866-1880, https://doi.org/10.1175/ 2009JPO4212.1.

McDougall, T. J., 1987: Neutral surfaces. J. Phys. Oceanogr., 17, 1950-1964, https://doi.org/10.1175/1520-0485(1987)017<1950: $\mathrm{NS}>2.0 . \mathrm{CO} ; 2$.

_ , and R. Feistal, 2003: What causes the adiabatic lapse rate? Deep-Sea Res. I, 50, 1523-1535, https://doi.org/10.1016/ j.dsr.2003.09.007

— and D. R. Jackett, 1988: On the helical nature of neutral trajectories in the ocean. Prog. Oceanogr., 20,153-183, https:// doi.org/10.1016/0079-6611(88)90001-8.

Pogliani, L., and M. N. Berberan-Santos, 2000: Constantin Carathéodory and the axiomatic thermodynamics. J. Math. Chem., 28, 313-324, https://doi.org/10.1023/A:1018834326958.

Theodorou, A. J., 1991: Some considerations on neutral surface analysis. Oceanol. Acta, 14 (3), 205-222.

Zachanoglou, E., 1973: Carathéodory's theorem on the Second Law of thermodynamics. SIAM J. Appl. Math., 25, 592-596, https://doi.org/10.1137/0125056. 